\title{
Philosophiques
}

\section{Exactitude et bavardage.: Gloses pour une opposition paradigmatique dans la philosophie autrichienne}

\section{Kevin Mulligan}

Volume 26, numéro 2, automne 1999

La critique de la raison en Europe centrale

URI : https://id.erudit.org/iderudit/004959ar

DOI : https://doi.org/10.7202/004959ar

Aller au sommaire du numéro

Éditeur(s)

Société de philosophie du Québec

ISSN

0316-2923 (imprimé)

1492-1391 (numérique)

Découvrir la revue

Citer cet article

Mulligan, K. (1999). Exactitude et bavardage.: Gloses pour une opposition paradigmatique dans la philosophie autrichienne. Philosophiques, 26(2),

177-201. https://doi.org/10.7202/004959ar
Résumé de l'article

La philosophie autrichienne, depuis Bolzano jusqu'à Musil et Wittgenstein en passant par Mach et la tradition brentanienne, est marquée par une obsession singulière : la clarté et la précision. Quelques traits de cette obsession, en particulier la critique sévère des différentes formes de bavardage philosophique, sont décrits et situés par rapport à la culture autrichienne en général. Mais chaque vertu a son vice, et les vertus cognitives de la pensée autrichienne n'échappent pas à la règle. Quatre exemples de la pathologie de la précision sont analysés : Freud, Ehrenfels, Weininger et le dernier Husserl (le philosophe allemand). 


\title{
Exactitude et bavardage.
}

\author{
Gloses pour une opposition paradigmatique \\ dans la philosophie autrichienne \\ KEVIN MULLIGAN \\ Université de Genève \\ Kevin.Mulligan@lettres.unige.ch
}

\begin{abstract}
RÉSUMÉ. - La philosophie autrichienne, depuis Bolzano jusqu'à Musil et Wittgenstein en passant par Mach et la tradition brentanienne, est marquée par une obsession singulière : la clarté et la précision. Quelques traits de cette obsession, en particulier la critique sévère des différentes formes de bavardage philosophique, sont décrits et situés par rapport à la culture autrichienne en général. Mais chaque vertu a son vice, et les vertus cognitives de la pensée autrichienne n'échappent pas à la règle. Quatre exemples de la pathologie de la précision sont analysés : Freud, Ehrenfels, Weininger et le dernier Husserl (le philosophe allemand).
\end{abstract}

\begin{abstract}
Austrian philosophy from Bolzano, Mach and the Brentanian tradition to Musil and Wittgenstein is characterised by an obsession : clarity and exactness. Some features of this obsession, in particular the harsh criticisms of different types of philosophical blethering, are described, as is their place within Austrian culture. Each virtue has its vice and the cognitive virtues of Austrian thought are no exception. Four examples of the pathology of exactness are analysed : Freud, Ehrenfels, Weininger and the later Husserl (the German philosopher).
\end{abstract}

\section{L'exactitude à l'intérieur et à l'extérieur de la philosophie}

La production artistique et théorique de la monarchie danubienne, surtout dans les trente dernières années de son existence, se caractérise entre autres par une recherche de clarté et d'exactitude, recherche que l'on ne remarque pas seulement comme un thème qui réapparaît sans cesse, mais qui se révèle surtout dans l'effort d'être exact.

J'aborderai ce thème en deux temps : j'aimerais premièrement rappeler le caractère répandu et central du thème de l'exactitude dans la monarchie danubienne. J e m'occuperai ensuite d'un courant théorique peu connu, mais qui fut néanmoins très influent à l'intérieur de la philosophie (ou plutôt des philosophies universitaires) et qui étudia de manière intense l'opposition entre philosophie exacte et philosophie inexacte. Les travaux de Franz Brentano, ainsi que ceux de ses élèves et « héritiers », sont à bien des égards de véritables paradigmes de la philosophie moderne. Ils préfigurent ainsi la « philosophie analytique », surtout en regard des « préceptes » d'exactitude qu'ils ont établis et de leur description des variétés de la philosophie inexacte. Pour Brentano, mais aussi par exemple pour Robert M usil et pour des philosophes autrichiens plus tardifs tels que L udwig W ittgenstein, la thématisa- 
tion expresse de l'exactitude et de son contraire, le bavardage, était une préoccupation théorique centrale. II en est de même pour leur grand prédécesseur Bernard Bolzano, le « maître de la clarification » (H ugo Bergmann) et pour Ernst $\mathrm{M}$ ach ${ }^{1}$, leur grand contemporain.

M algré l'importance de l'opposition dans l'ensemble du spectre de la création artistique ainsi que la place tenue par sa partie constitutive, nommément l'activité philosophique des brentaniens et de M usil, cette opposition ne saurait être traitée de manière adéquate que moyennant une taxo-nomie encore inédite de l'histoire des idées. Aussi, j'y contribuerai par quelques indications, pour ensuite exposer deux choses : d'une part, je m'occuperai des formes de bavardage contre lesquelles se sont élevés un si grand nombre d'artistes et de penseurs autrichiens et par rapport auxquelles ils se sont eux-mêmes définis. D'autre part, j'aimerais souligner une sorte de bavardage spécifique à I'A utriche et qui, vraisemblablement, ne se produit que là où la conscience de I'opposition entre exactitude et bavardage est déjà affinée. Selon un mot de Goya, le rêve ou le sommeil ferait naître des monstres à la raison. Au moyen des quelques exemples qui suivent, je tenterai de montrer qu'à cette raison, dont l'histoire des idées de la monarchie danubienne nous livre tant d'exemples, le sommeil engendre des monstres typiquement autrichiens.

$M$ ais venons-en d'abord à cette affirmation selon laquelle l'étude de l'exactitude en général et de celle de l'expression en particulier se retrouve partout dans le monde austro-hongrois des idées. Prenons comme exemple les années 1900 : dans sa préface à la « Fackel », Kraus écrivit en 1899 qu'il projetait un « assèchement du vénérable marécage de phrases », qu'il espé rait « réduire le pesant sérieux du verbiage ». Cette expression de K raus concerne le jugement d'événements politiques. Comme on le sait, les moyens de cet assèchement furent progressivement l'analyse et le désassemblage de la forme et du contenu des phrases.

Presque parallèlement, $M$ usil projette un roman dans lequel l'auteur est un « vivisecteur psychique»: «M onsieur le vivisecteur. $M$ a vie : les aventures et errances d'un vivisecteur psychique au début du vingtième siècle » (cf. Kraus : «psychologische Seziermethode »).

En 1900 précisément, K limt peint le « Panneau » ou fresque de plafond «Philosophie » pour I'Université de Vienne. Un débat violent s'entame aussitôt entre les professeurs, les uns reprochant à K limt sa représentation de «pensées floues par des formes floues » (une critique à laquelle adhère K raus), les autres tentant, commel'historien del'art Wickhoff, de démontrer que les préjugés idéologiques et esthétiques des professeurs étaient précisément ce qui les empêchait de voir ce que K limt « représentait de manière très claire $»^{2}$. En 1904, Bertha Zuckerkandl parle de la «Vision» de Klimt

1. Voir M ulligan, 1986, ainsi que M ulligan et Smith, 1986.

2. Voir Schorske, 1979, p. 232 et suivantes. Les idées déterminantes de Wickoff en histoire de l'art, prises en considération à la défense de K limt, ont été dével oppées plus avant par Riegl (1901), et popularisées par Worringer (1908). 
comme « représentant une lutte pour la vérité, pour le soleil vivifiant des connaissances scientifiques $»^{3}$.

Déjà en 1898, I'architecte autrichien A dolf Loos expose l'opposition entre ornement et exactitude de la façon suivante :

Plus le niveau d'un peuple est bas, plus celui-ci est dépensier dans son ornement, dans sa parure. L'Indien recouvre entièrement d'ornements chaque objet, chaque barque, chaque pagaie, chaque flèche. Reconnaître un mérite dans la paruren'est rien d'autre que de se mettre au niveau de l'Indien. L'Indien qui est en nous doit cependant être dépassé[...]. La recherche de la beauté dans la forme sans la faire dépendre d'ornements est le but auquel aspirel'humanité tout entière.

O u bien, comme il l'exprime plus tard : « L'absence d'ornement est un signe de force intellectuelle. ${ }^{4}$

L'économiste Eugen von Böhm-Baw erk, figure dominante de l'école marginaliste autrichienne, développe une analyse novatrice passant par I'absence d'ornement, que Schumpeter décrit de la manière suivante :

Böhm-Bawerk reformule le même contenu avec une perfection magistrale. II exploite l'essentiel avec assurance en ne faisant usage que de peu d'arguments, mais simples et décisifs. Avec une dépense minimale d'efforts, sans détours et avec la plus grande élégance, il rend justice aux théories, les unes après les autres. ${ }^{5}$

On pourrait donner d'autres exemples de l'opposition entre exactitude-analyse et phrase-ornement, et cela à d'autres époques que celle de 1900. J'aimerais cependant me pencher ici sur une théorie ou réflexion particulière, qui fut élaborée à Vienne, au sujet de l'opposition entre exactitude et verbiage.

II n'y a pas que les artistes qui adoptèrent I'opposition exactitude/ bavardage, mais également les philosophes. Les propos des membres du Cercle de Vienne à cet effet sont les plus connus, de même que leur condamnation de toute « métaphysique » - pour reprendre leurs propres termes comme un non-sens. Les membres du C ercle de Vienne s'appuyèrent pour la plupart sur leur interprétation du Tractatus de Wittgenstein, paru en 1921. Avant cela, et ensuite parallèlement à l'évolution du Cercle de Vienne dans les années vingt et trente, Robert M usil s'était occupé dans son roman et dans plusieurs essais de différents aspects théoriques de l'opposition exactitude/bavardage.

Encore un peu avant, dans le dernier quart du XIX ${ }^{\mathrm{e}}$ siècle, deux philosophes, Franz Brentano et Ernst $M$ ach, développèrent une série de réflexions sur la philosophie exacte et sur la pensée univoque par opposition à la spé-

3. Zuckerkandl, 1908, p. 91.

4. Loos, 1962, p. 65 et 288.

5. Schumpeter, 1952, p. 84 ; cité par Cacciari, 1978, dont le livre, même s'il n'est en aucune façon « exact », contient beaucoup d'inspiration. 
culation vague et les ingrédients superflus. Par ailleurs, les idées de Brentano sur ce sujet sont beaucoup plus détaillées que celles de $M$ ach et eurent une très grande influence sur ses élèves et héritiers. L'effet de la philosophie de Brentano et en particulier de ses idées sur la manière dont doit procéder la recherche exacte est un fait dans l'histoire des idées sur lequel pourtant, pour diverses raisons, on continue à fermer les yeux.

Kafka et M usil, H ofmannstahl et Schnitzler, M ahler et Schönberg, $M$ ach et Wittgenstein, Kraus et Kokoschka sont tous des noms connus. Le rôle que joue chez eux l'opposition entre clarté et ornement est également connu. M ais je ne peux pas présupposer de connaissances quant à l'effet de Franz Brentano et c'est pourquoi je renvoie à la figure 1, qui décrit la façon dont s'est propagée son influence depuis $V$ ienne sur l'ensemble de la monarchie, et comment elle a formé la philosophie et la science du XX ${ }^{\mathrm{e}}$ siècle.

Les élèves et héritiers de Brentano étaient tous très marqués par son opinion sur ce qu'est une philosophie exacte. En 1866 déjà, Brentano avait prononcé à Würzburg deux thèses d'habilitation, selon lesquelles : (1) la philosophie doit protester contre la répartition des sciences en sciences spéculatives et sciences exactes et la justification de cette protestation est son droit même d'existence, et (2) la vraie méthode de la philosophie n'est nulle autre que celle des sciences naturelles ${ }^{6}$.

Avec la défense brillante de ces thèses à Würzburg - où, à la grande joie de Brentano, on avait inscrit sur la porte de l'auditoire, dans lequel on philosophait d'ordinaire à la Schelling, le mot « fabrique de soufre » - et à Vienne (1874-1895), Brentano gagna un très grand nombre d'auditeurs passionnés ${ }^{7}$. Aussi, ce que Brentano voulait propager avec ce qu'il appelait «philosophie exacte » est ceci : (1) une taxonomie des genres de philosophies inexactes et exactes; (2) une méthode de philosopher qu'adoptèrent tous ses élèves ; (3) une doctrine de l'unité des sciences.

\section{Ad 1}

Présentée tout d'abord dans une conférence en 1895 et connue comme sa doctrine des quatre phases de l'histoire de la philosophie, la taxonomie de Brentano est très simple ${ }^{8}$ : il s'aperçut du fait qu'on pouvait observer à trois moments dans l'histoire de la philosophie une série de quatre phases. À la première phase, qui se caractérise par un intérêt purement théorique, se rattache A ristote et, au M oyen Âge, saint Thomas. Elle s'étend jusque dans les temps modernes de Bacon, D escartes et Locke à Leibniz. Suit alors, en trois phases, le déclin, qui se caractérise d'abord par un affaiblissement del'intérêt scientifique souvent en faveur de motifs pratiques - les Stoïciens, Duns

6. Brentano, 1929, p. 136.

7. Le contenu de ces leçons est présentement disponible : Brentano, 1982, voir à ce sujet M ulligan et Smith, 1985.

8. Voir Brentano, 1926. 
Figure 1

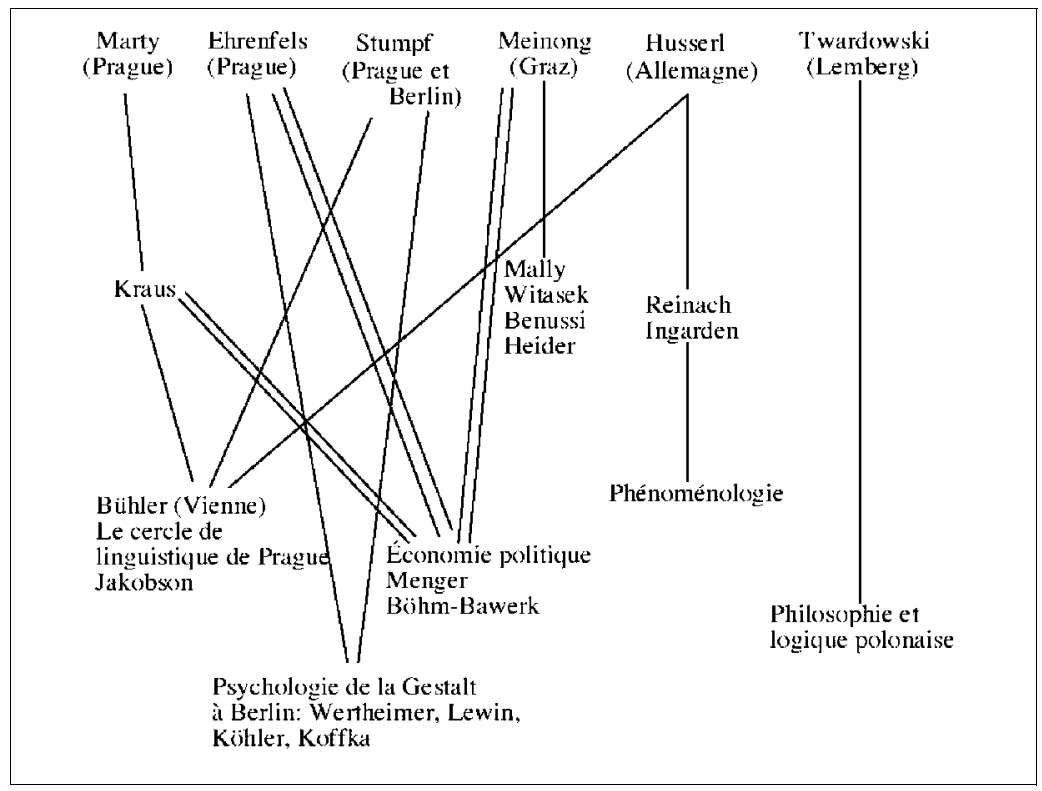

Scot, l'«A A ufklärung » (Voltaire, Wolff) - puis par le scepticisme - Sextus Empiricus, O ccam, H ume - et enfin par le mysticisme spéculatif - Plotin, $\mathrm{N}$ icolas de C use, Fichte, $\mathrm{H}$ egel.

La requête naturelle de vérité, retenue dans sa course par le scepticisme, perce par la force. Avec un zèle maladif, on revient à l'édification de dogmes philosophiques. Aux moyens naturels dont s'est servie la première phase, on en rajoute d'autres en inventant des moyens de connaissances tout à fait dépourvus de naturel, des principes sans le moindre discernement, des forces géniales, immédiatement intuitives, des élévations mystiques de la vie intellectuelle et bientôt, on se grise de la prétendue possession du plus sublime, des vérités dépassant tout ce qui est à la portée de l'homme. ${ }^{9}$

La taxonomie brentanienne de l'exactitude, du bavardage et des transitions entre les deux, taxonomie qu'il tenta de rendre plausible par des " considérations relatives à la psychologie culturelle», ne devait pas, bien entendu, contenir de prédictions. Les cours que donnait Brentano à Vienne sur ce sujet et qui se réfèrent nettement à la situation philosophique de cette époque faisaient tous preuve d'un espoir optimiste quant au commencement d'une nouvelle période de développement. Cependant, Brentano et presque tous ses élèves moururent convaincus que la période de clarté n'avait été que trop brève et que la décadence se manifestait à nouveau.

9. Brentano, 1929, p. 9. 
Ad 2

Brentano avait montré à ses héritiers une manière de philosopher que ceuxci adoptèrent et qui est, elle aussi, très simple : la philosophie doit rester incomplète. C'est plutôt l'examen détaillé de problèmes particuliers qui importe. Aussi, nous retrouvons ce principe de la philosophie par petits pas chez tous les héritiers de Brentano. À cet effet, l'une des premières grandes études de $M$ einong, Ü ber Annahmen (Sur les suppositions), s'étend sur trois cents pages et constitue l'examen d'un seul phénomène psychologique. II en est de même pour Twardowski et son Z ur L ehre von I nhalt und G egenstand der Vorstellungen. D 'une manière générale, les brèves dissertations et les études de thèmes étroitement délimités deviennent alors de rigueur. C'est également dans cet esprit que Ehrenfels fonda, en 1890, la psychologie de la Forme dans le cadre d'un exposé de 32 pages examinant simplement la question de savoir si une mélodie est une somme de sons. Q uant aux R echerches logiques de H usserl, dans les années 1900-1901, elles se composent de sept recherches sans lien direct entre elles ; comme l'écrivit H usserl lui-même ${ }^{10}$, il s'agit d'une « série de recherches systématiquement liées [...] mais non d'un livre ou d'une œuvre dans le sens littéraire ».

Cette prédilection marquée pour des attaques ou incisions partielles et ponctuelles, mais précises, se trouve justifiée par une idée que l'on trouve d'abord chez H usserl et $\mathrm{M}$ einong, mais dont le Tractatus donne la formulation la plus concise: «Une proposition peut sans doute être une image incomplète d'un certain état de choses, mais elle est toujours une image complète. ${ }^{11}$

Dit autrement, tant qu'une proposition ou une théorie satisfait à certaines exigences en ce qui concernela clarté et le sens, quel que chose demeure représenté, même si la connexion entre cet état de choses et d'autres reste vague. 0 mettre de considérer cela provoque, dans la phase spéculative de la philosophie,

l'apparition immédiate ou à la suite d'associations de pensées que l'on ne saurait appeler une médiation sensée [...] des affirmations les plus téméraires. On s'imagine pouvoir atteindre les hauteurs les plus inaccessibles; oui, on pense les avoir atteintes et c'est avec les suppositions les plus arbitraires que l'on comble les grandes lacunes de la connaissance ${ }^{12}$.

Le second élément constitutif de la méthodologie brentanienne est la nécessité de prendre comme point de départ une description scrupuleuse et sans conditions. II ne faut jamais confondre une description avec une explication génétique ${ }^{13}$. C eci représente en fait une revalorisation considérable du

10. Recherches logiques, vol. I, xi-xii.

11. Tractatus, 5.156.

12. Brentano, 1968, p. 56.

13. On peut caractériser de structurelle la distinction brentanienne entre le point de vue diachronique et le point de vue synchronique, ainsi que l'application de cette distinction à la 
rôle des exemples en philosophie ; on leur attribue trois tâches : ils servent premièrement à donner une signification aux termes et distinctions philosophiques et à fixer celle-ci. O $n$ donne leur sens aux concepts ou thèses en précisant les exemples appartenant à un ordre inférieur qui tombent sous leur application. Pour reprendre la métaphore préférée de H usserl : on doit pouvoir tout réduire en monnaie. D euxièmement, par une entente sur le contenu de certains exemples, il est possible de contrôler des thèses et hypothèses. Enfin, il est plus facile d'identifier les dimensions variables et constantes d'un phénomène au moyen d'une description détaillée ${ }^{14}$.

Depuis la «présentation d'un exemple concret de la naissance d'un désir » (« Vorführung eines konkreten Beispieles vom Entstehen eines Begehrens ») de Ehrenfels, qui allait servir de « substrat » à son analyse du désir, jusqu'à la « description naïve de la formation d'un sentiment » de $M$ usil ${ }^{15}$, cette stratégie restera un procédé constant dans les travaux philosophiques des héritiers de Brentano. H usserl, par exemple, peut se vanter, dans la préface de son premier livre Philosophie de l'arithmétique ${ }^{16}$, de n'avoir employé aucune terminologie qui n'ait été introduite à l'aide d'exemples ou de définitions. Lorsqu'il critique le livre Z ur Theorie der $\mathrm{N}$ aturwissenschaftlichen B egriffsbildung du néo-kantien Rickert, le reproche qu'il adresse à l'auteur - reproche que I'on retrouve aussi chez Brentano - est le suivant :

J'ai la conviction qu'une théorie fructueuse de la formation scientifique des concepts ne peut être qu'une théorie " par le bas », qui s'est constituée à partir du travail même sur les sciences naturelles. La théorie de Rickert se situe dans des constructions générales au point que l'on ne trouve pas un seul exemple dans tout le traité et que l'on n'en regrette même pas l'absence ${ }^{17}$.

Un autre élément de la méthode brentanienne est ce qu'on ne saurait mieux décrire que par le maintien conscient de l'attitude naïve de l'auteur d'une découverte. Brentano a caractérisé l'apogée de la philosophie exacte comme une époque où "I'on s'attaque à de nouvelles questions », où «s'approfondissent les hypothèses » et où « les questions se multiplient et $s^{\prime}$ interpénètrent $\gg^{18}$. D ans leurs analyses très détaillées du langage, du sentiment, de la volonté, de la préférence et de la valorisation, les brentaniens se montrèrent pourtant parfaitement conscients du fait que leurs problèmes et leurs solutions étaient nouveaux. Pour eux, cela faisait partie de l'activité philosophique que de faire des découvertes - si l'on avait de la chance. C'est

théorie du langage et à la psychologie. R iegl et Wickhoff - voir p. 2 du présent article et plus haut - développèrent simultanément l'idée d'une analyse formelle et synchronique des types stylistiques. L'élaboration d'une $\mathrm{G}$ renznutzenlehredescriptive autrichienne fut développée également comme une alternative à ladite « école historique».

14. Comparer sur ce point M ulligan, 1986.

15. Ehrenfels, 1897, p. 68 (titre) ; M usil, chap. 54 de L'homme sans qualités.

16. Page VII.

17. H usserl, 1897, maintenant dans H usserl, 1972, p. 147.

18. Brentano, 1895, maintenant dans Brentano, 1926, p. 8 et 10. 
ce regard-là que porte Ehrenfels sur sa découverte des qualités des formes et c'est ainsi que $M$ einong considère sa découverte - il parle de « contrée théorique inconnue » - des suppositions ; il s'agit là d'une attitude cognitivequi est centrale aussi bien pour la logique que pour l'esthétique. On peut encore citer la découverte de la tripartition acte/contenu/objet que fit Twardowski, ainsi que la découverte des états de choses comme étant ce qui rend vraies des assertions, comme étant le véritable objet du souhait et du désir, etc. Toutes ces découvertes sont expressément considérées comme la clarification de quelque chose que l'on avait omis jusqu'alors de reconnaître.

Cette attitude des brentaniens trouve sa meilleure expression dans une remarque de Bertrand R ussell concernant une découverte qu'il avait faite avec W ittgenstein : « aujourd'hui, nous avons trouvé une nouvelle bête pour notre zoo ${ }^{19}$.

Le dernier élément de la méthode brentanienne consiste en un usage abondant de définitions et d'arguments déductifs. Stumpf décrit : « Ce procédé qui nous vient de Brentano, respectivement d'A ristote, la préparation à la preuve directe par la disjonction totale de tous les points de vue possibles, et par la réfutation de tous à l'exception d'un seul. » 20

On retrouve ce procédé partout dans les travaux des brentaniens; l'argument rigoureux de quarante pages dans les R echerches logiques de H usser ${ }^{21}$ en fournit un bon exemple : H usserl y examine plusieurs interprétations possibles de la thèse selon laquelle tout acte est une représentation ou est fondé sur une représentation, et les utilise pour en tirer une preuve directe. Un autre exemple est donné par l'élimination faite par Ehrenfels d'une série de théories alternatives de la valeur.

Les philoso phes aiment à se faire passer pour de stricts penseurs déductifs, mais en réalité on ne trouve avant les brentaniens, dans les temps modernes, aucun groupe qui ait fait un emploi aussi systématique d'arguments déductifs (ou d'arguments en général) et d'exemples. Aurel Kolnai, I'un des derniers philosophes autrichiens, voit une connexion entre la philosophie autrichienne et la monarchie des $\mathrm{H}$ absbourg. Cette dernière serait :

plus qu'une nation, un état ou un empire, une administration immense et complexe, paternaliste et souvent tyrannique; un complexe administratif variable, incertain de sa fonction, mais essentiellement pluraliste et habitué à respecter de multiples façons toutes sortes d'intérêts, à pratiquer une espèce de laxisme tolérant qui était inconnu de l'état national. L'esprit administratif autrichien [...] éclairé et tolérant (surtout à partir de $M$ arie Thérèse) penchait davantage vers la constatation patiente et prudente de différences, de particularités que vers la persistance perfectionniste à trouver des solutions satisfaisantes et définitives. La philosophie, une plante plus tardive en Autriche (freinée par la forte présence du clergé) trouve un climat avantageux pour

19. Russell, 1918, dans Russell, 1956, p. 226.

20. Stumpf, 1924, p. 5.

21. § 32-43 
l'analyse descriptive par opposition aux constructions téméraires de systèmes, que ceux-ci soient idéalistes ou matérialistes. La philosophie autrichienne avait pour ainsi dire un arrière-plan administratif où la constatation prudente, les esquisses tactiques et le bricolage par petits pas avaient leur importance, contrairement aux réformes téméraires et à l'activisme concentré. ${ }^{22}$

De Brentano à Wittgenstein, on avait effectivement la conviction d'avoir découvert une méthode dont l'essentiel est un procédé par petits pas, mais que l'on peut décrire et démontrer de manière exacte et qui mène à une attitude envers la philosophie que l'on peut qualifier de ( « geschäftsmässig ») ; on a, comme disait Brentano, découvert le « bon procédé » 23 . Pour H usserl, la philosophie devait être un « domaine scientifique du type d'une entreprise » ${ }^{24}$. L'indication de W ittgenstein concernant I'une des méthodes de type mercantile qu'il avait introduite et dont il avait fait usage, était précédée par la remarque que son père avait été un homme d'affaires.

La méthode brentanienne de philosopher et sa taxonomie de l'histoire de la philosophie laissèrent à tous ses auditeurs une immense impression du jeune Freud, comme le montrent des lettres restées inédites (à Silberstein, son ami d'enfance) jusqu'à $\mathrm{M}$ einong et $\mathrm{H}$ usserl. Je ne citerai qu'un exemple parmi de nombreux autres, un témoignage d'Emil Utitz, camarade de classe de Kafka et participant à des discussions régulières de la philosophie brentanienne qui avaient lieu au Café Louvre à Prague : «J'étais enchanté de ce procédé : le caractère simple, sobre et rigoureux de l'absence de phrases, la reddition de comptes pleine de responsabilités par rapport à chaque concept par la mise en évidence de son origine dans l'expérience. »25

\section{Anti-bavardage}

A rgument, analyse et description donnent ensemble cette manière de penser que M usil appelait une « dissection » et Brentano une « anatomie microscopique (de la conscience) » ou une « description par analyse ». Aussi, non seulement les brentaniens, mais également le Cercle de Vienne - N eurath, Carnap, Schlick - prêchèrent tous les avantages de l'analyse et - pour employer une nouvelle fois une description qu'emploie R ussell au sujet de Wittgenstein - , l'évangile de l'exactitude (« the gospel of exactness »). Dans les deux cas, ces évangiles contiennent aussi bien I'analyse que la con-

22. Aurel Kolnai, un philosophe hongrois, enseigna en dernier lieu à Londres, où il mourut. En rapport avec l'explication de $\mathrm{H}$ elmut Bachmaier (dans ce volume, p. 237-270) à propos des $K$ affeehäusern littéraires, on devrait ajouter que dans ces $K$ affeehäusern viennois, on théorisait et philosophait. Kolnai écrivit en anglais dans un des cafés viennois les plus fréquentés le brillant diagnostic du phénomène nazi de The War against the West. Les économistes autrichiens se rencontraient au C afé Griensteidl, sans doute avant les jeunes Viennois.

23. Voir Brentano, 1982, p. 28 ; pour Wittgenstein, voir R hees, dir., 1981, p. 173 et p. 125.

24. Plessner, 1959, p. 15.

25. Utitz, 1954, p. 73. 
damnation de larges parties de la philosophie traditionnelle. Ces deux évangiles ne sont pourtant aucunement identiques. Les ressemblances qu'elles présentent peuvent être décelées par exemple dans une série d'articles critiques des années vingt et trente rédigés de part et d'autre par des anti-Spengler et des anti-H eidegger ${ }^{26}$.

En 1921, O tto N eurath, futur membre du Cercle de Vienne, écrivit son Anti-Spengler, dans lequel il se concentre sur la tendance qu'a Spengler dans son $D$ éclin de l'O ccident (U ntergang des A bendlandes) à « expliquer quelque chose de confus et de vague par quelque autre chose confuse et vague ». À la même époque paraît l'essai de M usil Esprit et expérience. Remarques pour les lecteurs réchappés du déclin de l'O ccident. Avec $M$ usil, la tradition autrichienne de la critique du langage en arrive à son point culminant. M usil donne même un lexique et une grammaire pour le discours de Spengler afin que tout le monde puisse reproduire semblable chose tout seul :

Les contraires vie et mort, perception et connaissance, forme et loi, symbole et formule [...] devenir/devenu, mouvement/repos, propre/étranger, âme/monde, direction/espace, temps/temps métrique, volonté/connaissance, destin/ causalité [...] physiognomie/systématique : voilà presque au complet les idées directrices à l'aide desquelles Spengler pratique des coupes dans un donné fondamental qui demeure essentiellement le même, de quelque côté qu'il l'aborde. [...] N 'importe qui peut reconstituer sa philosophie : le schéma en est des plus simples. II suffit d'adopter les prédicats "est en un certain sens", « devient en un certain sens », de négliger les différences secondaires dans la forme d'expression, puis de combiner chacun des concepts cités avec tous les autres, en affirmant la possibilité d'accord de tous les concepts cités en premier lieu dans chaque couple, comme de tous ceux cités en second lieu, et en niant toute possibilité de combinaison d'un concept situé en premier lieu avec un concept situé en second lieu; obéit-on docilement à ce schéma, on verra se réformer automatiquement toute la philosophie spenglérienne, et même quelque chose de plus. Exemples : la vie... est objet de perception, a une forme, est symbole, devenir, etc. Spengler verrait là le défaut de la rationalité ; je ne dis pas autre chose ${ }^{27}$.

En 1924 paraît le « Untergang des wissenschaftlichen D enkens. G losses zu Spenglers 'U ntergang des A bendlandes' » («Le déclin de la réflexion scientifique. G loses pour "Le déclin de l'O ccident" de Spengler ») ${ }^{28}$ du brentanien $\mathrm{O}$ scar Kraus, dans lequel il montre que:

II y a ici une mystique victime de la folie des grandeurs qui ose se faire passer pour la dernière philosophie de l'Europe de l'O uest et comme l'accomplissement de cette dernière, une spéculation comblée par l'esprit scientifique parce que même la compréhension la plus élémentaire pour la méthode d'une

26. N eurath, 1921, p. 71.

27. M usil, 1921 (trad. Philippe Jaccottet), maintenant dans M usil, 1983, p. 1052-1053.

28. Kraus, 1924, p. 18. 
recherche patiente lui fait défaut. De par cette particularité, il s'agit là d'une philosophie du déclin scientifique dans son auto-compréhension.

Dans son écrit sur « Les quatre phases de la philosophie », Franz Brentano avait caractérisé le stade suivant comme étant le niveau le plus bas du déclin philosophique: celui dans lequel la période montante de l'intérêt purement théorique et de la méthode conforme à la nature, tombée dans la platitude et affaiblie par des motifs pratiques après avoir provoqué un scepticisme étendu, est relayée par des « méthodes de connaissance dépourvues de naturel », " des forces géniales, immédiatement intuitives» et des " élévations mystiques de la vie intellectuelle». " J'ai toujours radicalement méprisé la philosophie pour son propre compte», a écrit Spengler. À cela, Kraus répond : " $A$ sa place, il met un répugnant méli-mélo de scepticisme, de philosophie de l'impérialisme et de mysticisme visionnaire grandiloquent... J amais la pensée scientifique ne sera-t-elle capable ici de suivre ${ }^{29}$. »

Parmi les diverses critiques que $K$ raus adresse à Spengler, ses remarques de l'ordre de la critique linguistique sont particulièrement intéressantes :

Quoi qu'il en soit, l'originalité de la philosophie spenglérienne de l'histoire consiste tout au plus en ceci, qu'elle dit des "cultures comme étant des substances » ce que Lasaulx dit des peuples, et ceci justement est une plate absurdité. Spengler prend une abstraction grammaticale "culture» qui, comme d'innombrables autres mots tels que "langage, économie, état, écriture », exprime en un concentré linguistique divers côtés saisis de manière conceptuelle qui se rapportent à des individus en action, il prend donc une fiction commode du point de vue linguistique, un raccourci, pour en faire une chose, un être, une substance. D'une fiction, il fait - selon des modèles bien connus - une hypothèse et hypostase ${ }^{30}$.

Comme M usil, Kraus veut critiquer l'expression de Spengler ; c'est ainsi qu'il cite, à titre d'exemple, plusieurs passages de Spengler :

Spengler dit que $[\ldots]$ « le véritable homme d'État est l'H istoire en personne » (Vol.2, p. 552) ; «Le noble est l'homme en tant qu'H istoire» (p.435); « La femme est $\mathrm{H}$ istoire » (p.405); « La femme en tant que temps est la raison pour laquelle il existe une histoire des États ». Désormais on ne sait vraiment plus : I'H istoire est-elle un homme ou une femme? ${ }^{31}$

On peut constater de semblables concordances entre les réactions de Carnap et du brentanien $\mathrm{K}$ raus lors de la conférence inaugurale de $\mathrm{H}$ eidegger en 1929, «Q u'est-ce que la métaphysique? » («Was ist M etaphysik? »), dans laquelle on trouve des phrases telles que :

Le N éant est originairement antérieur au « $\mathrm{N}$ on » et à la négation. C'est le $\mathrm{N}$ éant lui-même qui néantit.

29. Ibid., p. 45.

30. I bid., p. 47 ; ce type de critique du langage, mais aussi la défense de l'individualisme méthodologique et ontologique sont à trouver chez tous les héritiers de Brentano.

31. Ibid., p. 52. 
$\mathrm{Ce}$ mot de $\mathrm{H}$ eidegger est devenu un locus classicus. Encore avant $\mathrm{C}$ arnap, en 1930, K raus s'était intéressé à des philosophes qui prennent le $N$ éant au sérieux, tels que H eidegger et Rickert. En effet, commeil l'écrit : «À toute époque où resurgit le mystique, le $\mathrm{N}$ éant refait également surface et est à nouveau pris au sérieux. »

Kraus examine les mêmes phrases de $\mathrm{H}$ eidegger que Carnap et c'est ainsi qu'il clôt son étiologie : « Et si H eidegger dit que la science se ridiculise en ne prenant pas au sérieux le $\mathrm{N}$ éant, moi je réponds : toute science se ridiculiserait si elle le prenait au sérieux, à l'exception de la linguistique, de la critique linguistique et de la logique. ${ }^{32}$

Ce renvoi à la critique linguistique et à la logique nous mène cependant à une importante divergence entre les brentaniens et les positivistes. Kraus et Carnap soumettent les phrases de $\mathrm{H}$ eidegger à une analyse syntaxique et sémantique avant de les condamner comme bavardage. Ce procédé répandu chez les brentaniens signifie que la description d'une philosophie comme étant absurde a un sens précis et qu'il ne s'agit pas seulement d'une marque de refus. $M$ ais le genre et la fréquence de l'analyse employée par les brentaniens diffèrent énormément du genre et de la fréquence de l'analyse à laquelle adhéraient les positivistes. Les brentaniens furent les premiers philosophes modernes à prendre au sérieux la théorie des structures formelles, théorie qu'ils employèrent partout. $M$ ais contrairement aux positivistes, ils veillèrent toujours à consolider le caractère approprié d'une certaine analyse formelle dans un certain domaine d'application par des arguments psychologiques et ontologiques.

Or, cette disposition des brentaniens est presque totalement absente chez les positivistes. Cela se voit surtout dans l'attitude des uns et des autres envers la tradition: de larges portions de la tradition philosophique furent condamnées par les brentaniens et les positivistes, mais les brentaniens étaient cependant prêts à en faire la démonstration par des analyses détaillées de philosophes traditionnels - Kant, par exemple. Les divers termes, distinctions, arguments, etc. employés par Kant sont examinés séparément, avant que sa philosophie ne soit condamnée comme étant désespérément confuse. Ainsi, un caractère constant des critiques que les brentaniens adressent aux philosophies confuses est la constatation que, si l'on regarde attentivement les fondements descriptifs de philosophèmes traditionnels, si l'on tente d'indiquer les phénomènes d'ordre inférieur qui seuls pourraient donner un sens à ces philosophèmes, on ne trouve que trop souvent que ces philosophèmes flottent en l'air. Cette constatation est pourtant précédée la plupart du temps par la tentative de trouver un support pour les philosophèmes en question. Chez les positivistes cependant, on trouve rarement - abstraction faite d'exceptions telles que ladite analyse de Carnap - plus qu'une

32. Kraus, 1930, maintenant dans Kraus, 1934, p. 125. Carnap mentionne en l'approuvant l'attaque de Kraus. 
tentative cursive pour constater si une thèse de la philosophie traditionnelle est sensée ou non. En ce sens, on peut dire que la philosophie brentanienne n'est pas tant une forme préliminaire du positivisme que le positivisme est une caricature de la philosophie brentanienne.

Twardowski exprime sans doute l'opinion de tous les brentaniens lorsqu'il affirme qu'il n'est pas du tout nécessaire de s'occuper de philosophes confus. En philosophie, la liaison entre l'expression écrite et la pensée est si étroite, que « nous pouvons supposer sans autre façon que l'auteur qui n'est pas capable d'exprimer clairement ses pensées est incapable de penser clairement. C'est pourquoi il ne vaut pas la peine de se donner du mal à débroussailler ses pensées. ${ }^{33}$

Q u'il y eut, dans la monarchie danubienne, une longue et vive tradition de la critique philosophique du langage, et en particulier une tradition de I'A nti-K ant et del'A nti-H egel, est un fait connu. En 1798 déjà, dans un rapport adressé à la « Studien-R evisions-H ofkommission », le conseiller administratif Franz Karl Hägelin s'exprima contre l'enseignement de la philosophie kantienne: « Leibniz et Wolf n'introduisirent pas de nouvelle terminologie dans les sciences [...]. Kant [...] a provoqué l'effondrement, sans substituer quelque chose de solide ; il a introduit une terminologie toute neuve. Si I'on introduisait la philosophiekantienne, le résultat serait que l'on cause de choses obscures et que l'on ne comprenne rien aux choses essentielles. $»^{34}$

Cette tradition, que documenta récemment Roger Bauer, par exemple, dans son livre $D$ er I dealismus und seine $G$ egner in $O$ sterreich, atteignit son premier point culminant avec les critiques patientes et détaillées de Bolzano et l'élaboration de celles-ci par Prihonsky qui en fit Le nouvel Anti-Kant. A lors qu'on attribue à $K$ ant d'incessantes confusions, Bolzano parle du « bavardage schellingien-hégélien ». ${ }^{35} \mathrm{D}$ ans la même lancée, la tradition des soigneuses critiques de Kant que l'on omet souvent de considérer se poursuit avec les travaux de Brentano, de M arty et de Stumpf. L'expression de Brentano «À bas les préjugés! » ${ }^{36}$ signifie : à bas Kant!

Ehrenfels, par exemple, vit dans l'exigence kantienne de faire du caractère universalisable d'une action un critère de valeur de cette action, I'expression d'un « dogmatisme métaphysique et mystique ». M usil écrivit d'ailleurs quelques années plus tard:

L'impératif catégorique - et tout ce que l'on a tenu depuis pour un événement spécifiquement moral - n'est au fond qu'un détour, masqué de dignité bougonne, pour retrouver le sentiment. $M$ ais, ce faisant, ce que I'on met au

33. Twardowski, 1979, p. 2.

34. Hägelin, 1798, p. 7, 8 et 10.

35. Winter, 1966, p. 215.

36. Brentano, 1970, première partie. 
premier plan est quelque chose d'absolument secondaire et dépendant qui présuppose des lois morales au lieu d'en créer ; une expérience accessoire et nullement, il s'en faut!, l'expérience centrale de la morale) ${ }^{37}$.

Les brentaniens remplacèrent les théories générales sur des normes et la réduction implicite de l'éthique à l'action humaine par des examens minutieux dela structure de valorisations individuelles, pour savoir si celles-ci ont pour objet des actions ou autre chose. II s'agissait pour eux de trouver la relation entre valorisations, structures de préférence, sentiments et envie et non de prendre des positions éthiques idéologiques, si sublimes soient-elles. Ils voulaient montrer en particulier que de larges portions du conceptualisme traditionnel, dans le domaine de l'éthique par exemple, avaient vécu d'un régime d'exemples unilatéral. La multiplicité des valeurs ainsi que leur liaison à des actes de valorisation et aux faits de la vie affective auraient été négligées en faveur de normes et des actions qui s'y rattachent.

Plus encore que les avis partagés sur la philosophie scientifique exacte et sur la tradition philosophique, c'est une doctrine commune qui réunit les brentaniens et les positivistes. II est d'ailleurs frappant que dans les années quatre-vingt et quatre-vingt-dix déjà, Brentano ait élaboréà Vienne une doctrine de l'unité de toutes les sciences. ${ }^{38} \mathrm{C}$ ette doctrine représente les trois dernières parties de la philosophie exacte de Brentano. Dans une forme élaborée, elles sont normalement associées au programme du Cercle de $V$ ienne. $M$ ais, en fait, tous les brentaniens étaient partisans de la thèse selon laquelle il n'y a pas, pour la méthode, de différence entre sciences naturelles et sciences humaines. $D$ ans les deux cas, on a affaire à des descriptions, des explications structurelles, des explications causales et à la construction d'hypothèses causales falsifiables. Ce programme fut défendu avec véhémence par Brentano et par ses héritiers, surtout contre un flot de discours philosophiques venant d'Allemagne - de Dilthey et Spranger jusqu'à $H$ eidegger - dans lesquels on postulait d'étranges entités chargées de sens et une méthode de compréhension sui generis s'y rattachant. À cet effet, Kraus écrit en 1929 :

À nouveau il se forme aujourd'hui un peu partout des suites à ces philosophèmes (de la philosophie spéculative et mystique). Ces philosophies contemporaines qui croient devoir se rattacher à la phase spéculative et romantique oublient que justement ce fut elle qui provoqua la dépréciation et la dérision de toute philosophie aux yeux des chercheurs scientifiques. On tente parfois de montrer que ces Romantiques étaient sans doute égarés en ce qui concerne la recherche scientifique, mais qu'ils auraient été novateurs et

37. Le « caractère redoutable de la morale » dans M usil, 1983, p. 1003 (trad. Philippe J accottet). Voir Prosa, p. 898. L'œuvre centrale des brentaniens, qui justifie l'affirmation sur ce plan, est celle de M einong, 1917. Sur le rapport avec Stumpf, M einong, M usil et particulièrement St. Baley, voir M ulligan, 1988.

38. Brentano, 1929 ; voir aussi Stumpf, 1907. 
exemplaires pour les sciences humaines. La méthode des sciences humaines serait en effet fondamentalement différente de celle des sciences naturelles. ${ }^{39}$

Il y a une grande ironie dans le fait que la défense du programme de l'unité des sciences ait conduit chez les brentaniens et les positivistes à des résultats très différents. Les deux philosophies voulaient contribuer aux sciences. À cause de leur compréhension du concept de structure $\mathrm{e}^{40}$, laquelle s'orientait sur le modèle du concept de structure tel qu'il est représenté dans la logique moderne des relations, et à cause des tendances physicalistes et behavioristes, les positivistes ne pouvaient pas vraiment avoir des effets très positifs sur ce qu'on appelle les sciences humaines. Les brentaniens par contre, qui avaient une toute autre théorie de la structure, qui, elle, respectait aussi bien les relations ontologiques que logiques, contribuèrent de façon décisive à la formation de la linguistique structurelle, de la psychologie de la Forme et eurent une fructueuse interaction avec certaines branches de l'économie politique autrichienne (M enger, Böhm-Bawerk, V. W ieser ${ }^{41}$ ).

J 'ai évité jusqu'à présent d'aborder plus précisément les thèses philosophiques des brentaniens. J'aimerais cependant mentionner ici trois de leurs idées - qui ne font pourtant aucunement partie des acquis les plus importants de ces philosophes, ceci à cause de la ressemblance avec d'autres idées qui furent influentes en A utriche-H ongrie à la même époque et parce qu'on omet souvent de prendre en considération cette ressemblance dans la littérature relative à l'histoire des idées de la monarchie. En fait, les brentaniens consacrèrent presque toutes leurs énergies philosophiques à deux domaines : celui de la psychologie descriptive et celui de la théorie des relations structurelles. Il s'agit là d'ailleurs de deux domaines qui avaient été passablement négligés dans la philosophie de langue allemande depuis Kant ${ }^{42}$. Aussi, ils considérèrent la psychologie descriptive comme l'application d'une théorie de la structure à des phénomènes linguistiques et psychologiques. N ous allons ici nous intéresser à trois thèses de cette psychologie descriptive. (La psychologie était, on le sait, une parole magique pour les penseurs et artistes autrichiens ; Thomas $M$ ann a d'ailleurs souligné le degré d'importance de l'observation intérieure dans le roman autrichien).

A. Grâce à l'influence de certains arguments de Brentano, le jeune H usserl, Stumpf et Ehrenfels étaient tous d'avis qu'il n'existait pas de I ch et qu'il n'y avait donc pas non plus de conscience de soi ou de « relation sujetobjet ». Cette thèse, sous la forme quel'on trouve chez M ach - « Lelch ne peut pas être sauvé » - eut une grande influence sur de nombreux articles en A utriche. Les brentaniens entreprirent alors de défendre la thèse contre des

39. Kraus, 1929, p. 150-151 (la remarque de l'éditeur de Brentano, 1929); voir particulièrement Kraus, 1928, maintenant dans Kraus, 1934, Bühler, 1927, Funke, 1927.

40. Sur ce concept de structure, voir Smith et M ulligan, 1982.

41. Cf. notre figure $\mathrm{n}-1$.

42. On ne trouve pas encore aujourd'hui de traduction française convenable pour les termes « philosophy of mind » et « analytic metaphysics ». 
objections éventuelles, c'est-à-dire de penser jusqu'au bout la déconfiture du Ich - comme le dit Ehrenfels en s'appuyant sur H öfler. ${ }^{43}$

$B$. Tous les phénomènes psychologiques sont conscients, mais on ne peut pas les observer - ainsi, il n'existe pas de description de la vie intime. Bien que l'on puisse observer des objets extérieurs, on n'est conscient de ses propres états mentaux qu'au moment même où ceux-ci sont vécus. Ces états et ces actes sont encastrés dans des relations causales et structurelles insaisissables dans leur ensemble. Les observations sont soumises à la volonté, et si je réussis à observer ma colère, celle-ci disparaît. L'évidence ou la conscience que nous avons par rapport à ce qui, à chaque instant, se passe en nous, n'est - pour employer une métaphore - qu'îlot de clarté. C'est pourquoi notre connaissance des phénomènes psychiques repose sur l'observation de phénomènes extérieurs comme les actions et sur l'emploi des signes. Le fait que et la manière dont nous vivons les choses sont définis par des déterminants et des motifs inconscients. ${ }^{44}$

On trouve de semblables raisonnements aussi bien chez $\mathrm{M}$ usil que chez Kafka - tous deux héritiers (éloignés) de Brentano. M usil écrivit d'ailleurs :

La psychologie appartient au domaine de la raison et la diversité des faits qui lui sont propres n'est pas non plus infinie comme nous l'apprend la possibilité d'existence de la psychologie comme science empirique. II n'y a que les motifs psychiques qui soient d'une diversité incalculable et la psychologie n'a rien à voir avec ceux-ci45.

\section{Et Kafka écrit ${ }^{46}$ :}

II n'est jamais possible de remarquer toutes les circonstances qui affectent l'ambiance d'un instant et opèrent même en elle [...]. Les distinctions ne prouvent qu'une chose : on a envie de se laisser influencer et de mettre en scène une vie artificielle en s'écartant le plus possible de soi-même, cachéderrière des préjugés et des fantaisies ${ }^{47}$.

C. Toutes les institutions sociales - l'argent, la loi, les échanges commerciaux, le langage - se sont constituées sans méthode, mais non au hasard, commeledit $M$ arty. II est clair quecettethèse del'individualismeontologiqueest

43. Ehrenfels, 1916, p. 63-64. Brentano défendait sans doute le je.

44. Brentano, 1874, p. 40 et suivantes, p. 272. Voir Rug et M ulligan, 1986.

45. Musil, 1983, p. 1029. L'intéressante contribution de $M$ usil à la psychologie descriptive - dans les D ruckfahnenkapiteln de son roman (voir la note 37 ci-dessus) - est souvent mal évaluée, parce que l'on exagère l'influence de $M$ ach sur $M$ usil. Ce dernier était, du temps de sa dissertation, un « réaliste critique », comme Stumpf et les autres brentaniens. Voir mes remarques sur la traduction anglaise de sa dissertation (M usil, 1982) et L'homme sans qualités, p.1026 et 1045.

46. Je reviens sur la tentative, que les distinctions brentaniennes soulèvent entre la conscience intime et l'impossibilité d'observer nos vécus, pour expliquer les repères narratifs centraux dans l'œuvre de Kafka. Voir par exemple Smith, 1981.

47. Kafka, Tagesbücher, 1976, p. 248. 
étroitement liée au point B et à l'hostilité mentionnée plus haut des brentaniens par rapport à toute espèce de discours pompeux portant sur « la société », etc.

\section{La pathologie de l'exactitude}

A près cette esquisse des conséquences du désir d'exactitude à l'intérieur de la philosophie autrichienne, j'en arrive maintenant à un phénomènequi ne peut être compris que par rapport à l'arrière-plan de cette étude, peut-être devraiton même dire une obsession de l'exactitude.

II existe une tension étrange qui se manifeste surtout dans la production philosophique et intellectuelle de la monarchie danubienne. Cette tension occupe une place importante dans I'histoire des idées de cette époque. II s'agit d'une tension entreles « préceptes autrichiens » tels qu'ils sont exposés autour de 1900 dans les textes philosophiques et scientifiques les plus importants - les préceptes de l'analyse, de l'exactitude, de la description prudente « par le bas », de la clarté, de l'absence de toute forme d'absurdité et de théorie pompeuse «par le haut » - et, d'autre part, diverses sortes de transgressions de ces préceptes. De telles transgressions peuvent simplement consister dans le fait qu'une théorie ou un discours manque d'observations ou de descriptions nécessaires d'un domaine de phénomènes ; mais elles peuvent aussi être l'expression d'un penchant pour des constructions conceptuelles ou pour le bavardage. Comme nous l'avons vu, cette opposition et cette tension sont thématisées à l'intérieur de la philosophie. A ussi, j'aimerais ici décrire quatre cas qui exemplifient cette tension : Freud, Ehrenfels, Weininger et le second $\mathrm{H}$ usserl.

Freud lui-même, ainsi que bon nombre de ses lecteurs, font toujours resurgir un doute concernant le caractère scientifique ou non scientifique de sa manière de procéder et de ses idées. C e doute ou cette question ne se forma et n'eut d'intérêt pendant si longtemps qu'à cause del'indéniable multiplicité des descriptions et des analyses précises que fait Freud, à cause des hypothèses qu'il établit et poursuit scrupuleusement et à cause de ses processus d'argumentation ramifiés - en définitive, donc, à cause de ce qui paraît être la rigueur de son procédé. Freud lui-même veille bien à ce que le lecteur soit impressionné par cette rigueur méthodique. Un aperçu de l'accueil réservé à Freud dans les pays de langue allemande, tel qu'il a récemment été exposé par Brodthage et $\mathrm{H}$ offmann ${ }^{48}$, révèle le nombre de reproches tels que « dépourvu de méthode » ou « non-scientifique » qui lui sont adressés. Les travaux de Freud contiendraient des tentatives d'explication circulaires ou des explications qui, pour d'autres raisons, seraient inacceptables du point de vue formel ; ils consisteraient en réifications de concepts et contiendraient des portions de théorie déjà dépassée. Bon nombre de ces reproches sont faits par Karl Bühler dans son livre D ie K rise der Psychologie, mais l'auteur y

48. Brodthage et H offmann, 1981. 
exprime aussi son admiration pour la « prodigieuse lutte de Freud pour la clarté philosophique », en particulier dans ses derniers livres. ${ }^{49}$ Pour notre sujet, l'intérêt se situe surtout au niveau de l'attitude de W ittgenstein par rapport aux travaux de Freud. D'une part sa grande admiration, d'autre part la conviction qu'il ne s'agit en aucun cas de science, mais bien plutôt des réflexions d'un artiste génial ${ }^{50}$.

Le second exemple de l'alternance entre exactitude et bavardage concerne le double de Freud, $\mathrm{O}$ tto Weininger. Weininger fait un usage abondant de théories, arguments et matériaux provenant de la biologie, de la philosophie, de l'anthropologie, de la psychologie sexuelle, etc. ${ }^{51}$, mais ceci pour prêcher l'idéal masculin du célibataire purifié. Son œuvre majeure, G eschlecht und Character, constitue un énorme revers au monde des idées autrichien orienté vers l'exactitude contre le bavardage. Ce n'est pas un hasard si un apologiste de Weininger - et patient de Freud - fait la distinction entre une partie agréable, une partie sérieuse et une partie haineuse de son livre, cette dernière partie ne pouvant « en aucun cas avoir de rapport avec la science ou la philosophie ${ }^{52}$. II est surprenant de voir que, de tous les grands noms qui se montrèrent impressionnés par Weininger - Wittgenstein, Kraus, Canetti, Schönberg, Gomperz et, d'après Lessing ${ }^{53}$, M ach, Simmel, Bergson, $\mathrm{H}$ öfler et $\mathrm{M}$ authner - , pratiquement aucun ne sut donner les raisons de son admiration.

J'en arrive à présent à mon troisième exemple de bavardage d'un niveau supérieur, celui de Christian von Ehrenfels : c'est lui qui découvrit les qualités des Formes et il est aussi le premier philosophe qui réussit à développer en détail une théorie des valeurs tout à fait naturaliste à la Spinoza. À côté des brillantes analyses de ce philosophe, psychologue et théoricien des valeurs, nous trouvons dans l'œuvre d'Ehrenfels plusieurs esquisses étranges du domaine de la philoso phie de l'histoire, de la philosophie des religions, de type cosmologique et de l'ordre de la réforme sexuelle. Leur trait commun n'est pas le simple fait d'ignorer les « préceptes autrichiens », mais la tentative ratée d'incorporer et de transgresser ces derniers.

$M$ ax Brod fait d'ailleurs une description impressionnante des effets qu'eut sur lui cette tension telle qu'elle se présente dans plusieurs travaux d'Ehrenfels :

$M$ algré toute la subtilité qui le caractérisait, Ehrenfels [...] se situait toujours dans les dangereux parages du démentiel. C'est ainsi du moins que cela semblait après un premier aperçu furtif. S'intéressait-on davantage à Ehrenfels et à son système, que ne tardait pas à se manifester la solide logique de sa pensée. $M$ ais

49. Bühler, 1927, p. 164 ; Bühler était fortement influencé par les brentaniens. Voir M ulligan, 1986 et $1986 \mathrm{~b}$

50. Wittgenstein, 1966, p. 44.

51. Comme A. Janik l'a exposé de façon détaillée dans J anik, 1982.

52. Swoboda, 1911, p. 41.

53. Lessing, 1930, p. 198. 
elle aussi et elle surtout pouvait provoquer l'effroi et renforcer parfois (peut-être à tort) l'impression de démence qui s'était imposée déjà au départ ${ }^{54}$.

M uni de tous les moyens dont dispose un bon brentanien, Ehrenfels se lance dès 1902 dans tout un flot d'exposés dans lesquels il défend les vertus de la polygamie pour les hommes doués ${ }^{55}$. Ses idées sur la réforme sexuelle sont très soigneusement élaborées et portent aussi bien sur des raisonnements socio-darwinistes que sur le fonctionnement des « maisons de femmes » qu'il aimerait voir s'organiser.

Dans son célèbre essai L'Allemand comme symptôme (D er deutsche M ensch als Symptom), M usil se penche sur ce cas particulier de non-sens et de naïveté que présente un projet comme celui d'Ehrenfels. C'est justement parce que la tradition, les formes d'organisation et les institutions sociales donnent une forme à I'homme qui auparavant en est dépourvu - et M usil, peut-être par allusion aux observations de $\mathrm{N}$ ietzsche sur la mnémotechnique sociale (dans la G énéalogie de la morale), parle à cet endroit d'une « cruauté monstrueuse et inévitable » - que la propagande efficace d'un réformateur à la Ehrenfels présuppose exactement ce qui la rendrait superflue.

Essayons en second lieu, à l'aide d'un exemple, de concevoir l'amorce d'une époque nouvelle. II y a parmi nous beaucoup de polygames clandestins; mais officiellement, et jusque devant leur conscience, nombre d'entre eux prônent la monogamie (selon la recette de la règle tolérante aux exceptions qui régit toute notre morale). II s'agit là d'un état déjà labile, et rien n'empêche d'imaginer que tout d'un coup (dans des circonstances qui affaibliraient les églises et les partis conservateurs), la propagande de quelques détracteurs résolus du mariage entraîne une modification du code dont on pourrait vraisemblablement attendre des innovations d'ordre spirituel vraiment profondes. $M$ ais « on pourrait imaginer » signifie notamment: il faudrait [...], il faudrait que l'émancipation de la femme eût fait de grands progrès, que la socialdémocratie fût résolue à prendre au sérieux son ancien idéal, ce qui implique derechef des modifications de la situation économique dont on ne peut mesurer l'ampleur, et l'on ne peut prévoir si la presse étouffera le mouvement dans l'œuf ou l'appuiera: autrement dit, le commencement d'une époque dépend de mille hasards. $M$ ais si toutes les conditions favorables étaient réunies, les innovations d'ordre spirituel ne seraient peut-être pas au rendezvous pour finir : car les « réformes » ne rendent pas I'homme autre qu'il n'est, sauf si elles vont dans le sens d'un large courant d'énergie spirituelle qui dépend, à son tour, d'un million de hasards. Coïncidence non légale de nombreux faits. O ù elle fait défaut, la propagande reste un phénomène absolument négligeable $\mathrm{e}^{56}$.

54. Brod, 1979, p. 209. Brod était I'auteur, avec Felix Weltsch, d'une recherche intéressante sur le problème des concepts vagues - c'est-à-dire inexacts. Cette recherche appartient clairement à la tradition des brentaniens, et tout particulièrement à celle de la psychologie descriptive martyienne (Brod et Weltsch, 1913).

55. Sur ce point, et sur la relation entre Freud et Ehrenfels, voir Rug et M ulligan, 1986.

56. M usil, 1983, p. 1370-1371 (trad. Philippe Jaccottet). 
L'intérêt et le flair de M usil pour les limites entre exactitude et bavardage sont tout à fait comparables à la capacité d'un Brentano de diagnostiquer le non-sens philosophique comme tel. M on dernier exemple sera le philosophe Edmund H usserl. Dix à quinze ans après avoir écouté à Vienne les cours de Brentano (1884-85), et suivi des études à $\mathrm{H}$ alle en compagnie de Stumpf, le plus ancien élève de Brentano, $\mathrm{H}$ usserl, avait déjà écrit deux grandes œuvres : sa Philosophie de l'arithmétique (1891) et l'un des plus grands livres de la tradition philosophique, les Recherches logiques de 1900-1901.

Les R echerches sont peut-être l'œuvre la plus importante qu'aient produite les brentaniens. On oublie toujours qu'elle fait partie intégrante de la tradition autrichienne, parce qu'on la lit dans l'optique du second H usserl, ou pire encore, dans l'optique du second $\mathrm{H}$ usserl et de son assistant $\mathrm{H}$ eidegger. Les R echerches reprennent des arguments et des distinctions que I'on trouve sans exception chez Brentano et les autres brentaniens, et c'est avec beaucoup de rigueur que H usserl les développe. Tous les traits de la philosophie exacte quej'ai cités jusqu'à présent sont parfaitement exemplifiés dans cette œuvre ${ }^{57}$.

A utour de 1906, il seproduisit pourtant quelque chosequi devait changer fondamentalement la nature de l'activité philosophique de $\mathrm{H}$ usserl. Alors qu'il avait, comme je l'ai dit, refusé au début toute forme de Ich ou de soi-même en dehors de la relation structurée des expériences vécues, il lui arriva néanmoins un jour, dans les dix premières années de ce siècle, d'apercevoir son I ch ${ }^{58}$. II semble que cet événement doive beaucoup au fait qu'il avait, lui, le brentanien, commencé à prendre beaucoup plus au sérieux K ant et les néo-kantiens.

En très peu de temps, H usserl fut perdu pour le monde de la philosophie exacte. Soit, ceci est le destin de nombreux philosophes dès le départ. Le cas de $\mathrm{H}$ usserl est inhabituel dans la mesure où il put se servir de ses précédentes découvertes. Le lecteur remarque comment il se sert de la rhétorique de l'exactitude, de la rigueur, de l'esprit scientifique d'une façon quasi inflationniste aux endroits où il a le moins de choses à dire.

À partir de 1907 et jusqu'à la fin de sa vie, Husserl produisit une série de textes qui n'ont pas tous été publiés, et dont font partie des œuvres influentes telles que les I dées I, les M éditations cartésiennes, L ogique formelle et transcendantale, la Crise des sciences européennes. Les élèves les plus anciens de $\mathrm{H}$ usserl, ceux de la monarchie danubienne comme Ingarden ou Kolnai, ou les phénoménologues comme Reinach et Daubert remarquèrent très rapidement que le philosophe $\mathrm{H}$ usserl avait beaucoup changé depuis les R echerches logiques. Ses écrits étaient « pesants », consistaient en de grossières ébauches, étaient « dogmatiques ». Ses élèves, déçus, restèrent pourtant fidèles à l'ancien réalisme et à l'idéal de la clarté59. Leurs descrip-

57. Voir M ulligan, 1986.

58. Recherche logique $V, \S 8$.

59. Voir Ingarden, 1959, p. $462 ; 1972$, p. 27. Voir aussi Linke, 1961, p. 63. 
tions des productions tardives de $\mathrm{H}$ usserl sont par trop clémentes. En fait, $\mathrm{H}$ usserl commet une erreur qui ressemble beaucoup à celle que les brentaniens avaient décelée chez de nombreux philosophes de la tradition. Il érige une énorme construction philosophique sur un fondement descriptif trop mince. À cet endroit, je ne peux qu'esquisser brièvement une critique qui va dans ce sens - une critique qui, pour cette raison, est aussi passablement injuste ${ }^{60}$. Le point de départ de Husserl en 1906 était une découverte qu'il avait faite en même temps que $M$ einong : la découverte des suppositions. Les jugements ne sont pas soumis à la volonté ; je ne peux pas décider de juger $p$. $M$ ais je peux très bien décider de supposer que $p$ est le cas, en considérant par exemple $p$ comme une hypothèse. Le phénomène des suppositions et son importance (entre autres) pour la logique furent immédiatement reconnus par des philosophes extérieurs à l'école de Brentano, tels que Bertrand Russell. Cette importance fut également anticipée partiellement par le logicien Frege et conduisit à la découverte de toute une série d'autres problèmes intéressants.

$M$ ais H usserl ne s'intéressait plus désormais à des problèmes de cette nature. Enchanté d'avoir aperçu son I ch - qui fut alors baptisé « le Ich transcendantal » ou la « Subjectivité transcendantale » - il pensa avoir découvert toute une série de sciences «nouvelles», « rigoureuses », « radicales», « profondes», « sérieuses», « fondamentales ». Il se trouve en effet que par les diverses « réductions » - de nature psychologique, eidétique, transcendantale, etc. - le l ch transcendantal est à même de découvrir diverses objectivités « constituées». A ussi, il faut comprendre toutes ses diverses « réductions » selon le modèle de la supposition volontaire. Elles sont toutes d'une manière ou d'une autre analogues. Or, H usserl ne parvient jamais à identifier l'analogie déterminante, aussi peu d'ailleurs qu'il réussit à expliquer le sens de termes comme, par exemple, constituer. Ceci ne l'a pourtant pas empêché de consacrer plusieurs milliers de pages aux possibilités combinatoires de ces termes - dans un discours dans lequel littéralement toutes les roues tournent sans que quoi que ce soit ne tourne avec. Si I'on voulait suivre le très méchant exemple de $\mathrm{M}$ usil, il serait facile d'établir une grammaire et un lexique pour la production tardive de $\mathrm{H}$ usserl.

Ces textes présentent les caractéristiques suivantes : ils ne contiennent pas d'exemples qui pourraient, comme c'était le cas chez le premier H usserl, servir au contrôle de ses analyses. Stumpf écrivit d'ailleurs sur les I dées de H usserl qu'« on y regrette beaucoup l'absence d'exemples, mais là où on en trouve, ils sont véritablement propres à induire le lecteur en erreur ${ }^{61}$. Ces textes de $\mathrm{H}$ usserl ne contiennent ensuite presque aucun argument, contrairement encore à ses anciens travaux, et enfin, ses constructions sont structurellement très semblables aux constructions de l'idéalisme allemand, et en

60. Voir par contre M ulligan, 1986.

61. Stumpf, 1939, p. 189. 
particulier à celles de Fichte - un fait qui, Iorsqu'il l'apprit, a tout de même gêné H usserl.

On ne saurait finalement être surpris du fait que le vieux Brentano, déçu dans son espoir que l'exactitude s'installerait en philosophie, parle de $H$ usserl comme du plus grand " sécessionniste » philosophique d'entre ses héritiers, de «Husserl et son kantisme du XX ${ }^{e}$ siècle ${ }^{62}$.

J'aimerais mentionner enfin une hypothèse intéressante qui pourrait peut-être expliquer comment $\mathrm{H}$ usserl a pu aussi rapidement et d'une façon aussi convaincue traverser presque toutes les étapes entre exactitude et bavardage. Cette hypothèse repose sur un vieux cliché selon lequel la mauvaise philosophie a quelque chose en commun avec la poésie ${ }^{63}$. Dans une monographie sur la phénoménologie et l'expressionnisme, Ferdinand Fellmann a tenté de montrer que la philosophie tardive de $\mathrm{H}$ usserl a de nombreux traits propres à l'expressionnisme littéraire. II montre comment, dans les deux cas, on utilise des formules toutes faites telles que « la vraie réalité », « la réalité réelle », et qu'il s'agit souvent d'amplifier « le sens de la réalité et de la possibilité ». Le style des I dées, dit Fellmann, est expressif et non descriptif.

La philosophie tardive de H usserl est-elle l'entreprise d'un artiste ou d'un mystique? Il est pratiquement certain que H usserl a voulu enseigner à ses lecteurs une nouvelle façon de voir les choses. L'hypothèse de Fellmann est en outre étayée par la lettre qu'écrivit $\mathrm{H}$ usserl à $\mathrm{H}$ ugo von $\mathrm{H}$ ofmannsthal après une rencontre avec le poète. II y écrit que sa méthode phéno-ménologique - qui est un « bénéfice durable » - est proche de cette position dans laquelle nous met « votre art comme étant une position purement esthétique quant aux objets qui y sont représentés et au monde environ-nant $»^{64}$.

Brentano, M arty, Stumpf, mais aussi Wittgenstein étaient des personnes profondément contemplatives. D ans leurs écrits, ils parviennent pourtant à nous donner une idée de ce qu'est la recherche de la clarté absolue - pour employer une expression favorite de W ittgenstein et des brentaniens - , sans pour autant passer outre les limites de ce que l'on peut clairement exprimer, puisque cette transgression serait même répréhensible. $M$ ais il n'est pas lieu d'aborder ici les ramifications de l'histoire de l'éthique de l'exactitude, de la valeur de l'univocité dans la production artistique et philosophique de la monarchie danubienne.

62. Brentano, 1966, p. 317 (lettre de 1916).

63. Voir la position de Carnap (1931) en opposition à celle de $\mathrm{H}$ eidegger et $\mathrm{N}$ ietzsche, ce dernier ayant dû être plus honnête en habillant ses idées artistiquement et non scientifiquement.

64. H usserl, 1907, dans Friedrich, dir., 1968, p. 111-112. K. H amburger a comparé de manière intéressante la poésie de Rilke à la méthode phénoménologique et la « vision des essences » de H usserl. 


\section{Bibliographie}

Baley, St., U eber U rteilsgefühle, Lernberg, 1916

Bauer, $R$., La réalité, royaume de $D$ ieu, études sur I'originalité du théâtre viennois dans la première moitié du XIX ${ }^{\mathrm{e}}$ siècle, M ünchen, 1965

- - , D er Idealismus und seine G egner in Ö sterreich, Heidelberg, Winter 1966

Brentano, F., D ie Vier Phasen der Philosophie, Leipzig, M einer, 1926

- - , Ü ber die Zukunft der Philosophie, Leipzig, M einer, 1929

- - , Die A bkehr vom N ichtrealen, H amburg, M einer, 1966

- - , Versuch über die Erkenntnis, H amburg, M einer, 1970

- - , D eskriptive Psychologie, $\mathrm{H}$ amburg, M einer, 1982

Brod, M ., D er Prager Kreis, Frankfurt, Suhrkamp, 1979

Brod, M. et Weltsch, F., Anschauung und Begriff, Leipzig, Wolff, 1913

Brodthage, $H$. et Hoffmann, S., «Die Rezeption der Psychoanalyse in der Psychologie» dans Cremerius, J., dir., Die Rezeption der Psychoanalyse, Frankfurt, Suhrkamp, 1981, p. 135-254

Bühler, K., D ie K rise der Psychologie, Jena, Fischer, 1927

Cacciari, M ., K risis - Saggio sulla crisi dei pensiero negativo da $\mathrm{N}$ ietzsche a Wittgenstein, M ailand, Feltrinelli, 1978

Carnap, R., «Überwindung der M etaphysik durch logische Analyse der Sprache », dans E rkenntnis II, maintenant dans Schleichert, dir., 1975, p. $149-172$

Fellmann, F., Phänomenologie und Expressionismus, Freiburg, Alber, 1982

Funke, O., Studien zur G eschichte der Sprachphilosophie. Berne, Francke, 1927

H ägelin, F.K., « Bemerkungen über die G edanken, die Kantische Philosophie betreffend » (1798), dans Wotke, K., Ein Beitrag sur Geschichte des Kantianismus in Ö sterreich, Wien, 1903

$\mathrm{H}$ amburger, K., «Die phänomenologische Struktur der Dichtung Rilkes », dans H amburger, K., Philoso phie der D ichtung, Stuttgart, 1966

H usserl, E., A ufsätze und Rezensionen (1890-1910), Den Haag, Nijhoff, 1979

Ingarden, R., «Edmund H usserl zum 100. Geburtstag», Zeitschrift für philosophische Forschung, vol. 13, 1959, p. 459-463

- - « What is new in Husserl's Crisis? », Analecta Husserliana, vol. II, Dordrecht, 1972, p. 23-47

Janik, A., "Therapeutic Nihilism": How not to Write About Otto Weininger » dans Smith, dir., 1981

- - , Essays on Wittgenstein and Weininger, Amsterdam, Rodopi, 1985

Kolnai, A., "Brentano's Place in the H istory of Philosophy », manuscrit de cours non publié, 1972

Kraus, O., «Der Untergang des wissenschaftlichen Denkens (O swald Spengler) », H ochschulwissen, 1924, p. 45-52 
- - « Geisteswissenschaft und Psychologie» (1928), maintenant dans Kraus, 1934

_- , « Über Alles und N ichts » (1930), maintenant dans Kraus, 1934

- - «Selbstadarstellung», dans H. Schmidt, dir., Philosophie in Selbstdarstellungen, Leipzig, M einer, 1929

- - , Wege und A bwege der Philosophie, Prague, Clave, 1934

Lessing, T., D er jüdische Selbsthass, Berlin, 1930

Linke, P., N iedergangserscheinungen in der Philosophie der Gegenwart Wege zu ihrer Überwindung, M ünchen, Reinhardt Verlag, 1961

Loos, A., Sämtliche Schriften, vol. I, W ien/M ünchen, H erold, 1962

Meinong, A., Emotionale Präsentation (1917), maintenant dans la G esamtausgabe, vol. III, 1968, p. 283-476

M ulligan, K., «Philosophy, A nimality and Justice : K leist, Kafka, Weininger and Wittgenstein », dans Smith, dir., 1981, p. 293-312

- - , « Exactness, Description and Variation: How Austrian Philosophy was Done», dans N yiri, C., dir., Ö sterreichische Philosophie, Vienne, H ölder-Pichler, 1986, p. 86-97

- - «On Structure: Bühler's Linguistic and Psychological Examples», dans Esenbach, A ., dir., K arl Bühler's Theory of L anguage, Amsterdam, Benjamins, 1988a, p. 203-228

- - « M usil and the A nalysis of Emotions », manuscrit, 1988

Mulligan, K. et Smith, B., «Franz Brentano's O ntology of $M$ ind », Philosophy and Phenomenological Research, 1985, p. 627-644

- - « M ach and Ehrenfels : The Foundation of Gestalt Theory », in Smith, dir., Foundations of Gestalt Theory, M ünchen, Philosophia, 1988, p. 124-157

M usil, R ., «M oralische Fruchtbarkeit » (1913), « Geist und Erfahrung » (1921), « Der D eutsche M ensch als Symptom » (1923), dans M usil, R., Essays und Reden, Frise, A., dir., Row ohlt, 1983

N eurath, O., Antispengler, M ünchen, Callwey, 1921

Plessner, H ., H usserl in G öttingen. Göttingen, Vandenhoeck, 1959

Rhees, R., dir., Ludwig Wittgenstein. Personal Recollections, Oxford, Blackwell, 1981

Riegl, A., Spätrömische Kunstindustrie, Vienne, 1901

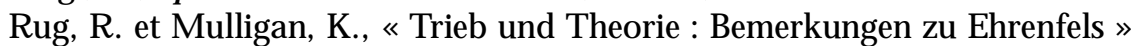
dans Fabian, R., dir., Das Leben und Werk von Christian von Ehrenfels, A msterdam, Rodopi, 1986

Russell, B., «The Philosophy of Logical Atomism » (1918), dans M arsh, R., dir., Logic and Knowledge, London, Allen \& Unwin, 1956

Schleichert, H ., L ogischer Empirizismus-W iener Kreis, M ünchen, Fink, 1975 Schorske, C., Fin-de-Siècle Vienna: Politics and Culture, Cambridge University Press, 1979

Schumpeter, J.A., Ten G reat Economists, Londres, Allen \& Unwin, 1952 
Smith, B., «Kafka and Brentano : A Study in Descriptive Psychology », dans Smith, B., dir., 1981, p. 113-160

- - , dir., Structure and G estalt: Philosophy and Literature in Austria$\mathrm{H}$ ungary and her Successor States, Amsterdam, Benjamins, 1981

Smith, B. et M ulligan, K., "Pieces of a Theory », dans Smith, dir., Parts and Moments. Studies in Logical and Formal O ntology, M ünchen, Philosophia, 1982

Swoboda, H ., O tto Weiningers Tod, Vienne, Deutlcke, 1911

Twardowski, K., Z ur Lehre vom Inhalt und G egenstand der Vorstellungen. Eine psychologische U ntersuchung, Vienne, H ölder, 1894

- - , " O n Clear and O bscure Styles of Philosophical Writing », dans Pelc, J., Semiotics in Poland, Dordrecht, Reidel, 1979

Utitz, E., «Erinnerungen an Franz Brentano », Wissenschaft zu der M artin$L$ uther U niversität $\mathrm{H}$ alle-W ittenberg, quatrième année, cahier 1,1954 , p. $73-90$

Von Ehrenfels, C., « Über Gestaltqualitäten», Vierteljahresschrift für wissenschafltiche Philosophie, 14, 1890, p. 242-292

- - , System der Werttheorie, vol. I et II, 1897/98, maintenant dans Werttheorie, M ünchen, Philosophia, 1982

- , «Die sexuale Reform», Politisch-anthropologische Revue, vol. II, 1904, p. $970-993$

- - , « Das M utterheim », Politisch-anthropologische R evue, vol. V, 1906

Winter, E., D er Böhmische Vormärz, H eidelberg, W inter, 1966

Wittgenstein, L., L ectures and Conversations on A esthetics, Psychology and Religious Experience, C. Barrett S. J., dir., O xford, Blackwell, 1966

- - , Wittgenstein und der Wiener Kreis, 1967

- - , N otebooks 1914-1916, 1979

Worringer, W., A bstraktion und Einfühlung, 1908

Zuckerhandl, B., Z eitkunst. W ien 1901-1907, Vienne/L eipzig, H eller et Cler, 1908 